\title{
Increasing Muscle Speed Drives Changes in the Neuromuscular Transform of Motor Commands during Postnatal Development in Songbirds
}

\author{
${ }^{\circledR}$ Iris Adam and ${ }^{\circledR}$ Coen P.H. Elemans \\ University of Southern Denmark, Department of Biology, 5230 Odense M, Denmark
}

Progressive changes in vocal behavior over the course of vocal imitation leaning are often attributed exclusively to developing neural circuits, but the effects of postnatal body changes remain unknown. In songbirds, the syrinx transforms song system motor commands into sound and exhibits changes during song learning. Here we test the hypothesis that the transformation from motor commands to force trajectories by syringeal muscles functionally changes over vocal development in zebra finches. Our data collected in both sexes show that, only in males, muscle speed significantly increases and that supralinear summation occurs and increases with muscle contraction speed. Furthermore, we show that previously reported submillisecond spike timing in the avian cortex can be resolved by superfast syringeal muscles and that the sensitivity to spike timing increases with speed. Because motor neuron and muscle properties are tightly linked, we make predictions on the boundaries of the yet unknown motor code that correspond well with cortical activity. Together, we show that syringeal muscles undergo essential transformations during song learning that drastically change how neural commands are translated into force profiles and thereby acoustic features. We propose that the song system motor code must compensate for these changes to achieve its acoustic targets. Our data thus support the hypothesis that the neuromuscular transformation changes over vocal development and emphasizes the need for an embodied view of song motor learning.

Key words: birdsong; motor control; rate coding; speed matching; spike timing; vocal muscle

\section{Significance Statement}

Fine motor skill learning typically occurs in a postnatal period when the brain is learning to control a body that is changing dramatically due to growth and development. How the developing body influences motor code formation and vice versa remains largely unknown. Here we show that vocal muscles in songbirds undergo critical transformations during song learning that drastically change how neural commands are translated into force profiles and thereby acoustic features. We propose that the motor code must compensate for these changes to achieve its acoustic targets. Our data thus support the hypothesis that the neuromuscular transformation changes over vocal development and emphasizes the need for an embodied view of song motor learning.

\section{Introduction}

Animal behavior results from system-wide interactions among nervous system, muscles, body, and the surrounding environment (Chiel and Beer, 1997; Nishikawa et al., 2007). During

\footnotetext{
Received Jan. 15, 2020; revised May 19, 2020; accepted May 21, 2020

Author contributions: I.A. and C.P.H.E. designed research; I.A. and C.P.H.E. performed research; I.A. and C.P.H.E. contributed unpublished reagents/analytic tools; I.A. and C.P.H.E. analyzed data; I.A. and C.P.H.E. wrote the paper.

The authors declare no competing financial interests.

This work was supported by the Danish Research Council (Grant DFF 5051-00195), the Carlsberg Foundation (Grant CF17-0949) to I.A.; and the National Institutes of Health (Grant 5R01-NS-099375-02) and the Novo Nordisk Foundation (Grant NNF170C0028928) to C.P.H.E. We thank Sam Sober for comments on an earlier version of the manuscript.

Correspondence should be addressed to Iris Adam at irisadam@biology.sdu.dk.

https://doi.org/10.1523/JNEUROSCI.0111-20.2020

Copyright $\odot 2020$ the authors
}

motor skill learning, the brain generates activation patterns causing behaviors with increasing reliability, while the body exhibits large changes due to growth and training (Adam and Elemans, 2019). Because of the long extent (months to years) of fine motor skill learning, little is known about how motor code changes and how the developing body contributes to behavioral changes. Recent work shows that small changes in biomechanics can cause behavioral state changes previously attributed to neural drive alone (Zhang et al., 2019). It is thus essential to include biomechanics when studying motor control (Nishikawa et al., 2007; Tytell et al., 2011) and especially over developmental timescales (Zhang and Ghazanfar, 2018).

An excellent model to study motor skill learning is vocal imitation learning in songbirds. In zebra finches, males learn how to sing early in life by first memorizing a tutor song, which they match acoustically through trial-and-error learning over 60 days 
(Tchernichovski et al., 2001). Vocal output is controlled by well characterized neural circuitry (i.e., the song system) that consists of the following two main pathways: (1) the anterior forebrain pathway (AFP), which is necessary to learn and maintain song (Scharff and Nottebohm, 1991); and (2) the motor pathway, which encodes the learned vocalizations and is needed throughout life to produce them. In the avian cortex, the motor pathway produces millisecond-scale, precisely timed complex sequences of motor commands (Chi and Margoliash, 2001; Hahnloser et al., 2002) that result in force trajectories by respiratory and superfast vocal muscles (Elemans et al., 2008; Elemans, 2014). The transform from spikes to force is often referred to as the neuromuscular transform (NMT; Zhurov and Brezina, 2006). During song, these force trajectories modulate pressure in the respiratory system (Srivastava et al., 2017) and the position and tension of vibrating structures in the sound-producing organ, the syrinx, thereby altering the vocal output (Riede and Goller, 2010; Elemans, 2014). Thus, via the NMT, motor commands of the song system directly drive the acoustic features of song.

The changes in song observed over the course of vocal imitation learning are typically attributed to changes in neural drive (Jarvis, 2004; Fitch et al., 2010). However, in this same period the syrinx and particularly the syringeal muscles that execute motor commands from the song system are also undergoing significant changes (Adam and Elemans, 2019). One change directly influencing the NMT is that force responses of syringeal muscles double in speed when stimulated by single spikes (Mead et al., 2017). The observed changes seem driven predominantly by use, suggesting that extensive training of syringeal muscles is essential to achieve their maximal speed (Adam and Elemans, 2019). However, in vivo muscle contractions underlying behavior are never elicited by single spikes, but by spike trains with variable interspike intervals (ISIs), and it remains unknown whether the observed changes in muscle speed over development functionally alter force profiles and thereby behavioral output.

Here we test the hypothesis that muscle properties relevant to the NMT change over vocal development in male songbirds. We investigated three critical NMT features related to activation by spike trains. First, we show that all speed parameters of twitch and tetanic contractions significantly increase over vocal development. Second, we show that forces are higher than expected by linear summation (i.e., supralinear summation) and that this effect increases over development. Third, we show that submillisecond spike timing can be resolved by syringeal muscles and that spike timing sensitivity increases with speed. Together, our data show conclusively that the NMT changes over vocal development. We propose that the song system must compensate for NMT changes to achieve correct song targets, such as pitch trajectories over syllables. Because motor neuron (MN) and muscle properties are tightly linked, our data allow for predictions on the boundaries of the yet unknown motor code that correspond well to cortical activity.

\section{Materials and Methods}

\section{Experimental animals}

All data presented here were collected in zebra finches (Taeniopygia guttata). Birds were raised in individual breeding cages together with their biological parents and siblings. In total, 80 zebra finches were used in this study (males, 55; females, 25). All animals were kept on a 12h light/ dark cycle and provided with food and water ad libitum. All experiments and procedures were performed in accordance with the Danish Animal Experiments Inspectorate (Copenhagen, Denmark).
To quantify speed-related contraction parameters of syringeal muscles over the course of vocal development, we extracted muscle fiber bundles from musculus tracheobronchialis dorsalis (DTB) of male syrinxes at the following four time points: at $25 \mathrm{~d}$ posthatch $(\mathrm{DPH})$, right before the start of the sensorimotor phase (Roper and Zann, 2006); at 50 $\mathrm{DPH}$, during plastic song; at $75 \mathrm{DPH}$, after the sensory phase has ended; and at $100 \mathrm{DPH}$, when song is crystalized and the birds are considered adults (Immelmann, 1984). We included a cohort of age-matched females for comparison. Sex was determined by dissection postmortem (25 DPH) or plumage (all other ages).

\section{In vitro muscle physiology}

Muscle fiber bundles were prepared and stimulated in vitro to record isometric force responses as previously described (Elemans et al., 2008; Srivastava et al., 2017). In brief, birds were killed by an isoflurane overdose, and the syrinx was exposed, isolated, and pinned down on SYLGARD-covered Petri dishes in cold, oxygenated Ringer's solution. Fiber bundles were obtained randomly from the left or right DTB. Depending on whether the tissue originated from males $(>30 \mathrm{DPH})$ or juveniles and adult females, fiber bundles consisted of a subsection of or the entire DTB. Muscle preparations were mounted in a temperaturecontrolled bath, which was continuously supplied with oxygenated Ringer's solution. The rostral end of the preparation was fixed to a force transducer (model 400A, Aurora Scientific) and the caudal end to a micromanipulator, which was used to control the length of the preparation (Fig. 1A). After mounting the muscle preparation in the bath chamber, it was allowed to rest for $20 \mathrm{~min}$. Muscle fibers were stimulated through the bath solution by a high-power follow stimulator (model 701C, Aurora Scientific) at constant voltage using platinum electrodes. This stimulator is designed for muscle work, and, with an output ranging from $0.4 \mathrm{~mA}$ to $1 \mathrm{~A}$, it guarantees we are stimulating muscle membranes directly and before activating the neuromuscular junction. Force and stimulation signals were low-pass filtered at $10 \mathrm{kHz}$ (EF120 BNC throughfeed low-pass filter, Thorlabs) and digitized at 20 or $40 \mathrm{kHz}$ (NI DAQ Board PCI-MIO-16E4, National Instruments). All stimulations were conducted at $39.0 \pm 0.1^{\circ} \mathrm{C}$, which is within the body temperature range of $38-44^{\circ} \mathrm{C}$ in zebra finches (Zann, 1996). As muscle contraction speed increases with temperature (Mead et al., 2017), we are thus slightly underestimating the speed of the muscle and all correlated parameters. All software to control the setup and record data were written in MATLAB (MathWorks; RRID:SCR_001622).

\section{Stimulation protocols}

First, we optimized stimulation amplitude (pulse width, $300 \mu \mathrm{s}$ ) for maximal force production. The typical stimulation amplitude in our setup was $16 \mathrm{~V}$. Second, we applied $100 \mathrm{~ms}$ pulse trains ranging from 100 to $800 \mathrm{~Hz}$ in $100 \mathrm{~Hz}$ steps, to determine the force frequency curve and the stimulus frequency at which maximal tetanic force was produced. Last, muscle length was optimized for maximal force for each preparation. The optimal length of fiber bundles was $4.55 \pm 0.45 \mathrm{~mm}$ (median $=4.5$ $\mathrm{mm}, N=45)$ and the cross-sectional area (CSA) was $0.10 \pm 0.16 \mathrm{~mm}^{2}$ (median $=0.05 \mathrm{~mm}^{2}, N=28$ ). CSA was calculated from dry weight, optimal length and density (Mendez and Keys, 1960). Combining our CSAs with published fiber densities in syringeal muscles (Christensen et al., 2017; Adam et al., 2020), our preparations consisted of $\sim 68$ muscle fibers. Assuming a constant distribution of superfast/fast fibers between muscles (Christensen et al., 2017), suggests our preparations consisted of 60 superfast and 8 fast oxidative fibers. Pulse width and optimal stimulation amplitude were kept constant for all stimulations within one animal.

A critical gap in our knowledge of the song system is that virtually no information is available about firing characteristics of the MNs during song. The output nucleus of both the AFP and motor pathway is the robust nucleus of the arcopallium (RA), which sends direct projections to the MNs located in the hypoglossal nucleus nXII (Nottebohm et al., 1976; Wild, 1993a, b). MNs in the tracheosyringeal part of nXII (nXIIts) innervate the syringeal muscles (Wild, 1997). Although multiunit recordings of nXIIts have been reported (Williams and Nottebohm, 1985; Otchy et al., 2019), no firing characteristics of single units in 
nXIIts that comprise the actual motor code exist, to the best of our knowledge. Thus, while neural coding in the song system upstream of the MNs is well described, virtually no information is available on the motor code controlling the vocal organ. High-quality neural data have been obtained from single units in the motor cortex (i.e., nucleus RA), which is directly driving the syrinx MNs in nXIIts, and we therefore used RA firing statistics to design our stimulation patterns. Over song development, RA firing activity develops from variable firing with a wide ISI distribution to sparse, bursty firing, that is precisely locked to song and has a very narrow ISI distribution (Leonardo and Fee, 2005; Ölveczky et al., 2011).

For each muscle preparation, we performed several stimulation experiments in series in the order described below (Fig. 1B). Each experiment consisted of several stimulation patterns (described in the section below for each experiment). Each iteration was started by a tetanic pulse train (100 for experiment 1 and $50 \mathrm{~ms}$ for other experiments) followed by $3 \mathrm{~min}$ of rest. Subsequently, all stimulation patterns of one experiment were applied in random order with a 2 min rest in between. Each experiment was then repeated for several iterations (specified below for each experiment) and patterns were rerandomized within each iteration.

Experiment 1: isometric stress and muscle speed. Each iteration consisted of a $100 \mathrm{~ms}$ stimulation at the frequency producing maximal force followed by four single twitches. The experiment was run for at least two iterations.

Experiment 2: supralinear force summation. We used four stimulation patterns consisting of two spike patterns with ISIs of 2, 4, 6, and $8 \mathrm{~ms}$, covering the range of ISIs most commonly observed in RA (Ölveczky et al., 2011). The experiment was run for at least three iterations.

Experiment 3: spike timing. To investigate the effect of spike timing on force responses, we designed a three-spike stimulation paradigm (based on the study by Srivastava et al., 2017), consisting of 29 stimulation patterns based on four different frequencies $(200,400,600$, and $800 \mathrm{~Hz}$ ). To introduce spike-timing changes without changing the overall rate, the middle spike was moved in small increments (range, $0.1-1 \mathrm{~ms}$ ) from its centered position toward the first or last spike. The stimulation patterns by frequency were as follows: at $200 \mathrm{~Hz}$, we used 11 stimulation patterns with ISIs between the first and second spike (ISI1) of $0.4,0.5,1,2,3,4,5,6,7,8,9 \mathrm{~ms}$. At $400 \mathrm{~Hz}$, we used seven stimulation patterns with ISI1s of $0.4,0.5,1,2,2.5,3$, and $4 \mathrm{~ms}$. At $600 \mathrm{~ms}$, we used six stimulation patterns with ISI1s of $0.4,0.5,1,1.66,2$, and $3 \mathrm{~ms}$. At $800 \mathrm{~ms}$, we used five stimulation patterns with ISI1s of $0.4,0.5,1,1.25$, and $2 \mathrm{~ms}$. The experiment was run for one iteration.

Experiment 4: refractory period. Stimulation patterns consisted of eight two spike patterns with ISIs ranging from 0.3 to $1.5 \mathrm{~ms}$ in $0.2 \mathrm{~ms}$ steps, and a single-pulse stimulation as control. The experiment was run for at least two iterations. Data were acquired on 13 additional animals and acquired directly after experiment 1 .

Data analysis

All data analysis was conducted in $\mathrm{R}$ ( $\mathrm{R}$ Project for Statistical Computing; RRID:SCR_001905).

Force decrease correction. During the experiments, the force output of the preparations decreased linearly due to dying muscle fibers. The above-described experiments took $3.71 \pm 0.57 \mathrm{~h}$ (maximally, $4.5 \mathrm{~h}$ ) and $1.2 \pm 0.39 \mathrm{~h}$, and the force decrease at the end of all experiments was
B

| $|\||\||\|| \mid \quad$ Twitch speed

| ||| || || || ||| | Supralinearity

| || ||| |||||||||||||||||||||||||| Spike timing Stop $\mathrm{t}<5 \mathrm{~h}$

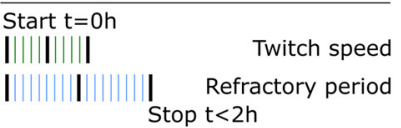

E

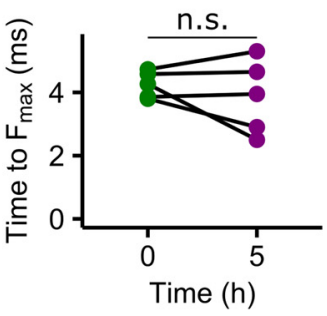

$\mathbf{F}$
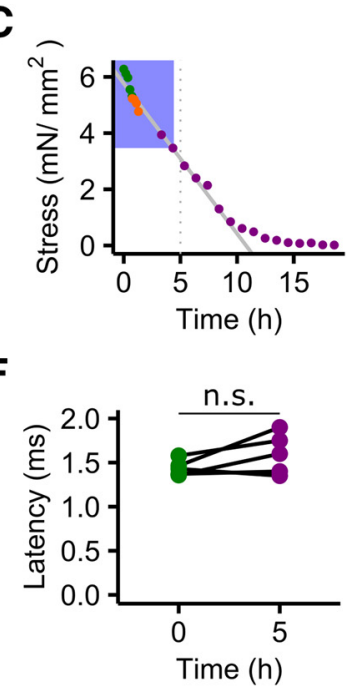

Time (h)

Figure 1. Muscle speed does not change over experimental time. $\boldsymbol{A}$, In vitro muscle physiology setup. Muscle fiber bundles were fixed to a force transducer (left) on the rostral end and to a motorized micromanipulator (right) on the caudal end. The (back) was continuously supplied with fresh oxygenated Ringer's solution. The red box indicates the inset show(solid gray line) over $>5 \mathrm{~h}$ (dotted vertical line). $\boldsymbol{D}-\boldsymbol{F}$, Data included in this study were all acquired within

$55.8 \pm 27.2 \%$ and $75.1 \pm 26.9 \%$, for experiment $1-$ experiment 3 and experiment $1+$ experiment 4 , respectively. To correct for this force decrease, we normalized all force data from our stimulation patterns over all experiments. For normalization, we used the linear regression between the maximal force of the $50 \mathrm{~ms}$ tetanic contractions, which were part of each iteration, and the experimental time. Typically, the force decrease over time was linear until force reached $15 \%$ compared with the start of the experiment (Fig. 1C), which was well past the end of the experiments included in this study.

Isometric stress. Stress was calculated as F/CSA of the muscle, where $\mathrm{F}$ is the force. The cross-sectional area CSA was estimated from the optimal (resting) length $L_{0}$, the dry weight (dry-wet conversion factor: 5 ) and density [1060 kg/m $\mathrm{m}^{3}$ (from the study by Mendez and Keys, 1960)] of the muscle fibers.

Muscle speed, single stimulations. The full-width at half-maximal (FWHM) force of single twitches was defined as the time from crossing $50 \%$ force increase to decrease, and is the same parameter as previously reported ( $t_{50-50}$; Elemans et al., 2004; Srivastava et al., 2017). The time to maximal force $\left(\mathrm{F}_{\max }\right)$ was defined as the time from stimulation to reaching the peak of the twitch. Latency was defined as the time from stimulation to the force signal crossing the mean plus five times the SD of a $50 \mathrm{~ms}$ segment of the force before stimulation. All values reported are the average of seven twitch contractions. The FWHM of two other syrinx muscles, VTB (ventral tracheobronchial) and musculus syringealis ventralis (VS), was extracted using previously published data from 12 (Mead et al., 2017) and 5 (Adam et al., 2020) additional animals, respectively.

Muscle speed, tetanic contractions. We fitted a mathematical model (Watanabe et al., 2010) to the fusion index-frequency curve (FFC) and force-frequency relation (FFR) using the $n l s$ function in $\mathrm{R}$ to extract the frequencies corresponding to $10 \%$ fusion (start of summed responses), $90 \%$ fusion $\left(f_{\text {fused }}\right)$, and $90 \%$ of maximal tetanic force $\left(f_{\text {Tet }}\right)$.

Supralinearity. We normalized the maximal force amplitude of all force traces to the $F_{\max }$ of the first peak of the $8 \mathrm{~ms}$ pattern within each iteration. A twitch model (Raikova and Aladjov, 2002) was fitted and used to calculate linear summations by adding two modeled twitches offset by the ISIs used for stimulation. Supralinearity was quantified as the ratio between the normalized and modeled $\mathrm{F}_{\max }$ values as well as the ratio of the area under the curve (AUC) of the normalized force data and 

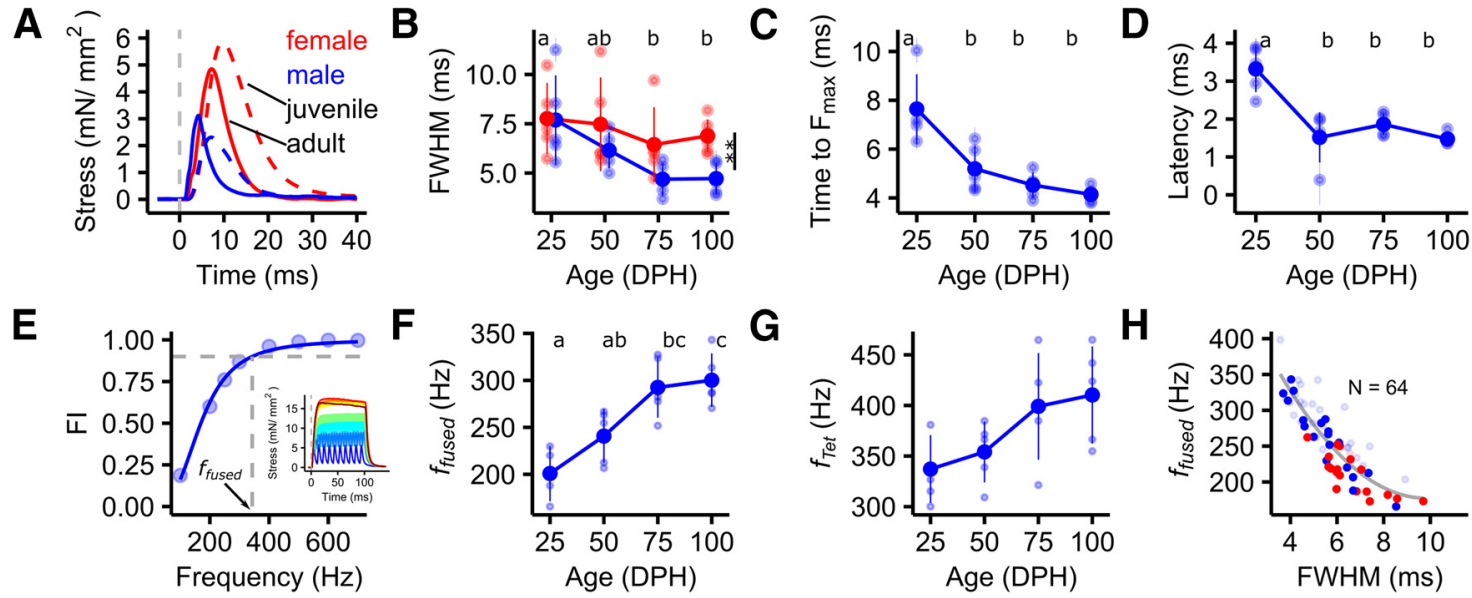

Figure 2. Contraction speed increases over development in male songbirds. $\boldsymbol{A}$, Twitch contraction speed was higher in adult males compared with juveniles or adult females, as illustrated by the narrower force peak. Examples of twitch responses of juvenile and adult male (blue) and female (red) zebra finch syrinx muscles. The dashed, vertical, gray line indicates the time point of stimulation. $B$, FWHM force decreased over vocal development in males, but not in females [linear mixed-effects model (LMM): FWHM $\sim$ sex + age $+(1 \mid$ animal); sex: df(1), $F=7.5$, $p<0.01$; age: $\mathrm{df}(3), F=3.9, p<0.05$; animal: $\mathrm{df}(1)$, likelihood-ratio test $(\mathrm{LRT})=835, p<0.001]$. C, $\boldsymbol{D}$, Time to $\mathrm{F}_{\text {max }}$ decreased significantly between 25 and $50 \mathrm{DPH}\left(\boldsymbol{C} ; \mathrm{ANOVA}\right.$ : $F_{s}=14.98$, $\mathrm{df}(3,16)$, relative $\mathrm{SE}(\mathrm{RSE})=0.91, p<0.001)$, as did the latency from stimulation to the force onset $(\boldsymbol{D} ; \mathrm{ANOVA:} F=16.5, \mathrm{df}(3,16), \mathrm{RSE}=0.48, p<0.001)$. $\boldsymbol{E}, \mathrm{A}$ mathematical model was fitted to the FFC and the FFR to extract the frequency when responses start to fuse [fusion index $(F I)=0.1]$, reach $f_{\text {fused }}$ and $f_{\text {Tet }}$, respectively. Depicted is an example of an FFC of an adult male. Crossing of the gray dashed line with the $x$-axis indicates $f_{\text {fused }}$. The inset depicts the force responses to stimulation with spike trains from 100 to $700 \mathrm{~Hz}$ (encoded by color) used to calculate Fl. $\boldsymbol{F}, \boldsymbol{G}, f_{\text {fused }}(\boldsymbol{F})$ and $f_{\text {tet }}(\boldsymbol{G})$ both increased significantly over vocal development $\left(f_{\text {fused }}:\right.$ ANOVA: $F=10.91, \operatorname{df}(3,15), \operatorname{RSE}=29.84, p<0.001 ; f_{\text {Tet: }}: \operatorname{ANOVA:} F=3.48, \mathrm{df}(3,16), \operatorname{RSE}=42.14$, $p=0.041)$. $\boldsymbol{H}$, FWHM and $f_{\text {fused }}$ are highly correlated $\left[N=64 ; \mathrm{LM}: \mathrm{f}_{\text {fused }} \sim \mathrm{FWHM}, p<0.001, \mathrm{RSE}=51.66\right.$, df(63)]. Solid points indicate data from preparations depicted in $\boldsymbol{B}, \boldsymbol{C}, \boldsymbol{D}, \boldsymbol{F}$, and $\boldsymbol{G}$, while transparent points are derived from additional preparations of other syringeal muscles, partially published in the study by Mead et al. (2017). Unshared lower case letters above plots indicate post hoc differences with a significance level $p<0.05$. ${ }^{* *} p<0.01$. A horizontal jitter was applied to data points in $\boldsymbol{B}$ for clarity.

the summation model. The AUC was calculated using the AUC function from the DescTools package (Signorell, 2019).

Spike timing. To quantify the variability introduced by changing spike timing, the coefficient of variation (CV) was calculated for the timing $\left(\mathrm{CV}_{\text {Timing }}\right)$ as well as for the amplitude $\left(\mathrm{CV}_{\text {Amplitude }}\right)$ of $\mathrm{F}_{\max }$ per stimulation frequency and animal for one iteration of the protocol.

Estimation of pitch changes. The fundamental frequency of sound is often called "pitch." Although pitch is a perceptual measure that is not always equivalent to fundamental frequency, we will refer to the fundamental frequency as pitch to avoid confusion between stimulation and sound frequencies. To illustrate the effect of spike-timing manipulations on sound production, we estimated the pitch changes that would occur if the entire VS would be stimulated simultaneously with the three-pulse patterns during sound production. We focused on VS, as it is well established that VS controls pitch (Goller and Suthers, 1996; Elemans et al., 2015; Srivastava et al., 2015). VS force was calculated for each animal by dividing the stress data by the CSA of VS $\left(1.05 \mathrm{~mm}^{2}\right.$; Adam et al., 2020). Pitch was calculated from force data using the force-pitch transformation (pitch $=26.6^{\star} \mathrm{F}_{\mathrm{VS}}$ ) of the VS (Adam et al., 2020). The average pitch difference per animal was calculated as the difference between the minimal and maximal pitch values per bird across all stimulation patterns of the spiketiming protocol. Translating our results acquired on DTB to VS is valid if stress and speed do not differ between the two muscles. We recently reported that stress generated by DTB and VS is not significantly different (Adam et al., 2020). Additionally, the FWHM ranges of DTB and VS are overlapping. We can thus interpret spike-timing results from DTB to VS.

Refractory period. $\mathrm{F}_{\max }$ values were extracted from all force responses and normalized to $F_{\max }$ of the control twitch within each iteration. To estimate the refractory period ( $t_{\text {Ref }}$ ), we fitted a log-logistic model to the normalized $\mathrm{F}_{\max }$ values using the $d r m$ function of the $d r c$ package (Ritz et al., 2015), as follows:

$$
f(x)=\frac{d-c}{\left(1+e^{(b(\log (x)-e)}\right)} .
$$

The $t_{\text {Ref }}$ was estimated as the ISI, where the normalized maximal force was $10 \%$ higher than of a single-twitch response using linear interpolation (Boërio et al., 2005).

Change of contraction speed over experimental time. Over the time needed to acquire all data for one preparation, muscle fibers were dying.
To exclude that this decrease differentially affected different fiber types (Christensen et al., 2017; Mead et al., 2017) with potentially different contraction speeds, we tested whether speed changed between the start of the experiment and $5 \mathrm{~h}$ of experimental time. We included data of preparations from adult males that was acquired between 4.5 and $5.5 \mathrm{~h}$ after the start of the experiment (Fig. $1 D-F$ ). The latency, FWHM and the time to reach $F_{\max }$ did not change significantly over $5 \mathrm{~h}$. We thus conclude that overall contraction kinematics are not affected by dying muscle fibers within the preparation.

Experimental design and statistical analyses

All data are reported as the average $\pm \mathrm{SD} ; N$ refers to individual animals. Statistical significance was accepted for $p<0.05$.

\section{Statistical tests}

Linear mixed-effects models with subsequent Tukey's post hoc comparison were used to assess statistical difference in the FWHM (Fig. 2B), $\mathrm{F}_{\max }$ (Fig. $3 B$ ), and supralinearity (Fig. $3 E$ ) datasets. Statistical design for each dataset can be found in the respective figure captions. ANOVA with subsequent Tukey's post hoc test was used to detect significant differences between age groups in the $f_{\text {fused }}$ (Fig. $2 F$ ), $f_{\max }$ (Fig. $2 G$ ), time to $\mathrm{F}_{\max }$ (Fig. 2C), and latency (Fig. 2D) datasets, and between stimulation frequencies and $\mathrm{CV}_{\text {Amplitude }}$ and $\mathrm{CV}_{\text {Timing }}$ (Fig. $4 \mathrm{~B}$ ). Linear models were used to test the correlation with $f_{\text {fused }}$ for FWHM (Fig. $2 H$ ), supralinearity (Fig. $3 F, G$ ), and $\mathrm{CV}_{\text {Amplitude }}$ and $\mathrm{CV}_{\text {Timing }}$ (Fig. $4 C$ ). Welch's $t$ test was used to test for sex differences in $t_{\text {Ref }}$ (Fig. $5 C$ ), and paired $t$ tests were applied to test changes in contraction speed over experimental time (Fig. 1D-F).

As the FWHM did not change significantly with developmental age in females, we did not analyze the female data for all further measures of muscle speed in the developmental dataset. To increase the range of muscle speed in our dataset quantifying supralinearity and the influence of spike timing, we included data from female DTB preparations.

All statistical comparisons were conducted in $\mathrm{R}$ ( $\mathrm{R}$ Project for Statistical Computing; RRID:SCR_001905).

\section{Results}

Contraction speed of syringeal muscles increases over vocal development in male songbirds

We measured isometric contraction speed of vocal muscles over song learning using the following two commonly applied 


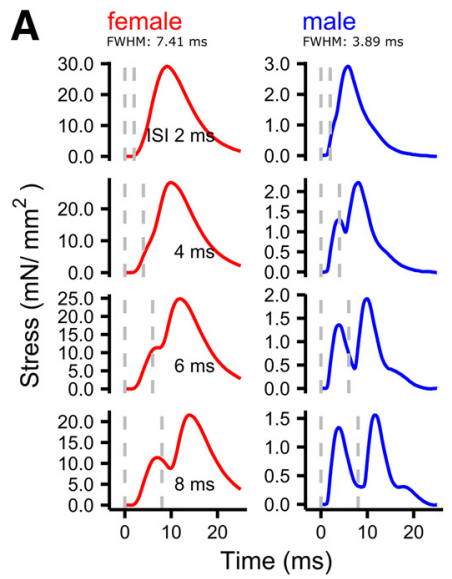

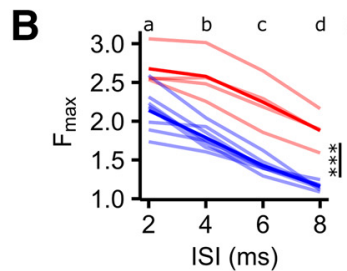
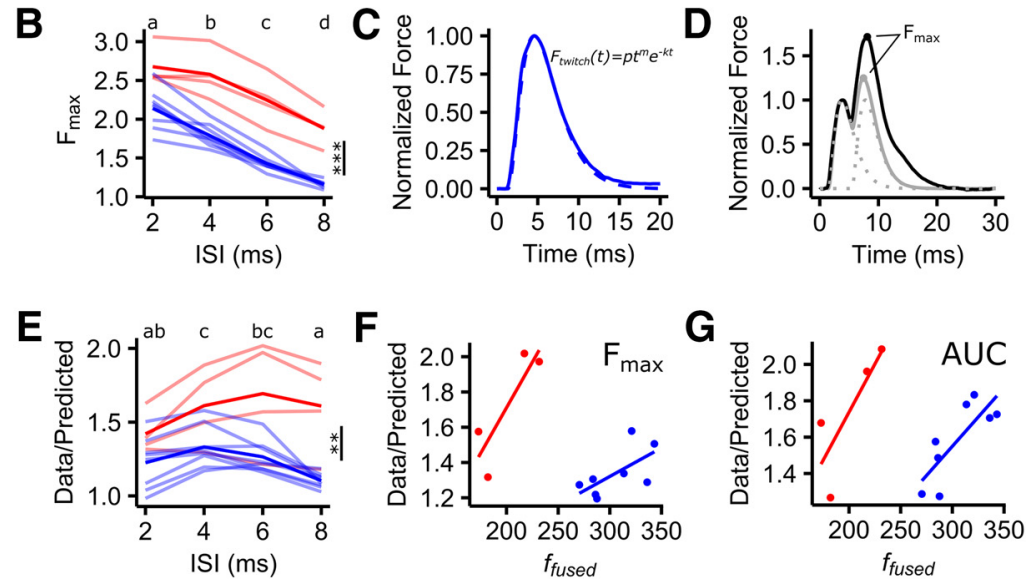

Figure 3. Force summation is supralinear and depends on contraction speed. $\boldsymbol{A}$, The shape of force trajectories changed dramatically with muscle contraction speed (male vs female) when driven by identical stimulation patterns. This is illustrated by examples of force responses of a female (red) and a male (blue) syrinx muscle to stimulations with two pulses with ISIs of $2,4,6$, and $8 \mathrm{~ms}$. The dashed, vertical, gray lines indicate the time points of stimulation. $\boldsymbol{B}$, Maximal force relative to a single twitch decreased with increasing ISI and was higher in males compared with females [linear mixed-effects model (LMM): normalized $\mathrm{F}_{\max } \sim \operatorname{sex}+$ ISI + (1 | animal); sex: df(1), $F=45.4, p<0.001 ;$ ISI: df(3), F= 102.3, $p<0.001 ;$ animal: df(1), likelihood-ratio test $(\mathrm{LRT})=16.3, p<0.001]$. Transparent lines represent individual preparations, and solid lines represent means. $C$, Twitch responses were fitted to a phenomenological model (Raikova and Aladjov, 2002). D, Recorded force responses (black) were compared with the summation model (gray) derived by summing two twitch models (dashed line). The amount of supralinearity was defined as the ratio between $F_{\max }$ or the AUC of the measured data and the summation model. $\boldsymbol{E}$, The amount of supralinearity depended on ISI and was higher in females compared with males (LMM: supralinearity sex + ISI + (1 | animal); sex: $\mathrm{df}(1), F=11.5, p<0.01$; ISI: $\mathrm{df}(3), F=5.2, p<0.01$; animal: $\mathrm{df}(1)$, LRT = 21, $p<0.001)$. 0n average, the maximum occurred at a lower ISI in males (4 ms) compared with females (6 ms). $\boldsymbol{F}, \mathbf{G}$, Maximal supralinearity increased with $f_{\text {fused, }}$, for both $\mathrm{F}_{\text {max }}\left(\mathrm{LM}\right.$ : ratio $\mathrm{F}_{\text {max }} \sim f_{\text {fused }}+$ sex; $f_{\text {fused }}$ : df( 2$), t=3, p<0.01$; sex: $\mathrm{df}(9), t=-4.3, p<0.01)$ and AUC (LM: Ratio AUC $\sim f_{\text {fused }}+$ sex, $f_{\text {fused: }}$ df( $(2), t=3.8, p<0.01$, sex: df(9), $\left.t=-4.1, p<0.01\right)$. Unshared lower case letters above plots indicate post hoc differences with significance level at $p<0.05$. $^{* *} p<0.01,{ }^{* * *} p<0.001$.

A Stimulation frequency
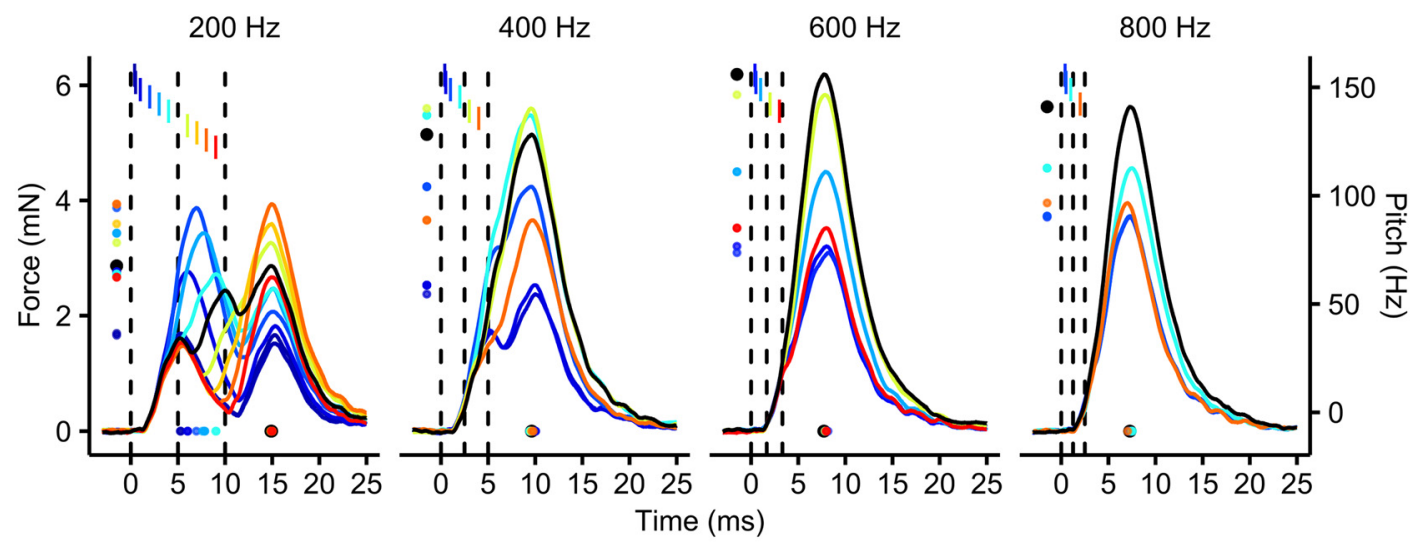

B

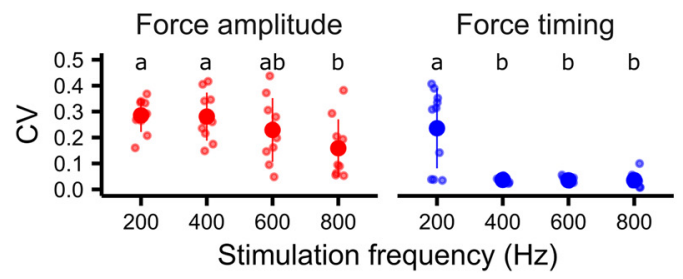

C

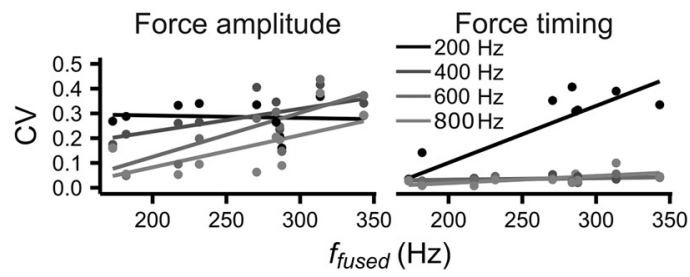

Figure 4. Submillisecond changes in spike timing affect the magnitude and timing of maximal force development in superfast syrinx muscles. $A$, Three-spike patterns with timing changes to the middle spike cause changes in both the timing and amplitude of force profiles as illustrated by example raw data of a male syrinx muscle (FWHM, $\left.4.45 \mathrm{~ms} ; f_{\text {fused }}, 284 \mathrm{~Hz}\right)$. Lines depict the normalized force response. Indicated are the timing (dots at bottom) and amplitude (left) of $F_{\text {max }}$. Vertical dashed bars indicate the timepoints of stimulation with regular ISls, and the colored bars indicate the stimulation time point of the middle spike when offset from the centered position. Color encodes the relative offset of the middle spike from its regular position. $\boldsymbol{B}$, The variability introduced by changes to spike timing depended on the base stimulation frequency and was generally higher at $200 \mathrm{~Hz}\left(\mathrm{CV}_{\mathrm{Amplitude}}, 0.29 \pm 0.06 ; \mathrm{CV}_{\text {Timing, }}, 0.24 \pm 0.15\right) \mathrm{Compared}$ with $800 \mathrm{~Hz}\left(\mathrm{CV}_{\text {Amplituder }} 0.16 \pm 0.11 ; \mathrm{CV}_{\text {Timing, }} 0.04 \pm 0.03 ; \mathrm{CV}_{\text {Amplitude: }}\right.$ ANOVA, $F=3.47, \mathrm{df}(3,36), \mathrm{RSE}=0.100, p<0.05, \mathrm{CV}_{\text {Timing: }}$ ANOVA, $\left.F=16.14, \mathrm{df}(3,36), \mathrm{RSE}=0.079, p<0.001\right) . \mathrm{C}$, $\mathrm{CV}_{\text {Amplitude }}$ significantly depended on muscle speed $\left(f_{\text {fused }}\right)$ at base stimulation frequencies of 600 and $800 \mathrm{~Hz}\left(600 \mathrm{~Hz}: N=10, \mathrm{LM}: \mathrm{CV}_{\text {Amplitude }} \sim f_{\text {fused }}, p=0.004, F=15.41 ; 800 \mathrm{~Hz}, N=10\right.$; $\left.\mathrm{LM}: \mathrm{CV}_{\text {Amplitude }} \sim f_{\text {fused }}, p=0.04, F=5.96\right)$, while $\mathrm{CV}_{\text {Timing }}$ did so at $200 \mathrm{~Hz}\left(N=10 ; \mathrm{LM}: \mathrm{CV}_{\text {Timing }} \sim f_{\text {fused }}, p=0.003, F=17.92\right)$. A horizontal jitter was applied to data points in $B$ for clarity. Unshared lowercase letters above plots indicate post-hoc differences with significance level $p<0.05$. 
A

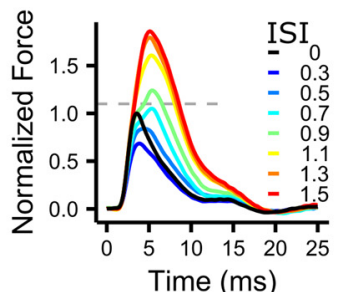

B

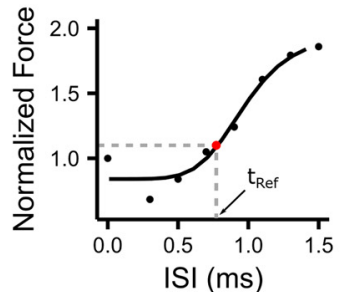

C

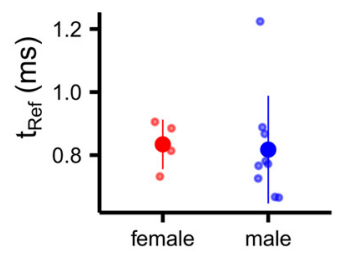

Figure 5. The refractory period of syringeal muscle fibers is submillisecond. $\boldsymbol{A}$, Force summation decreases with ISIs $<1 \mathrm{~ms}$ and eventually disappears completely as shown by example force traces of stimulations with short ISIs of a male syrinx muscle. Color encodes the ISI, and the dashed gray line indicates the threshold used to estimate $t_{\text {Ref. }} \boldsymbol{B}, t_{\text {Ref }}$ was calculated by fitting a log-logistic model and was defined as the ISI between two spikes, where the $F_{\max }$ is $10 \%$ higher than $F_{\max }$ of a single twitch. $\boldsymbol{C}, t_{\text {Ref }}$ of male and female DTB was not significantly different between males and females (Welch's $t$ test: $t=0.242, \mathrm{df}=10.87$, $p=0.81)$.

stimulation paradigms: (1) single stimulations yielding a single twitch contraction; and (2) stimulations with equidistant trains of spikes with varying frequencies $(100-800 \mathrm{~Hz})$, resulting in summed force responses (Fig. 2). At $25 \mathrm{DPH}$, FWHM of single twitches did not differ significantly between males and females and was $7.7 \pm 2.2 \mathrm{~ms}(N=5)$ and $7.8 \pm 1.8 \mathrm{~ms}(N=5)$, respectively. Over development, the speed of female DTB muscles did not change significantly and FWHM values stayed similar until $100 \mathrm{DPH}$ (6.9 $\pm 0.8 \mathrm{~ms}, N=6$; Fig. $2 B$ ). However, in strong contrast, male DTB muscles became steadily faster and finally doubled in speed with force FWHM almost halved to $4.7 \pm 0.8 \mathrm{~ms}(N=5)$ at $100 \mathrm{DPH}$, corroborating previous findings in another syringeal muscle (Mead et al., 2017). The time from stimulation to $F_{\max }$ (Fig. 2C) and latency from stimulation to force onset (Fig. 2D) also steadily increased over development but were already significantly different between 25 and 50 $\mathrm{DPH}$.

We measured three other muscle speed parameters by applying multiple equidistant stimulation trains, as follows: the stimulation frequency at which the force response (1) starts to summate, (2) is fully fused (i.e., $f_{\text {fused }}$ ), and (3) reaches $f_{\text {Tet }}$ (see Materials and Methods). The frequency at which twitches start to summate increased from $65.43 \pm 6.24 \mathrm{~Hz}$ at $25 \mathrm{DPH}(N=4)$ to $75.26 \pm 11.24 \mathrm{~Hz}$ in adult animals $(N=5)$. $f_{\text {fused }}$ increased steadily and significantly from $25 \mathrm{DPH}(201 \pm 29 \mathrm{~Hz}, N=4)$ to $100 \mathrm{DPH}$, reaching its maximum at $300 \pm 29 \mathrm{~Hz}(N=5$; Fig. $2 \mathrm{~F})$. Likewise, $f_{\text {Tet }}$ increased from $337 \pm 34 \mathrm{~Hz}$ at $25 \mathrm{DPH}(N=5)$ to $410 \pm 48 \mathrm{~Hz}$ at $100 \mathrm{DPH}(N=5$; Fig. $2 G)$. The $f_{\text {Tet }}$ was higher than $f_{\text {fused }}$ in all groups (seen by higher values in Fig. $2 F$ compared with Fig. 2G). This means that force still increased as a function of stimulation frequency even after the force responses were fully fused. This has also been reported in other motor systems (Kernell, 1995; Orizio et al., 2004). Interestingly, in our large dataset of 64 animals we found that force FWHM accurately predicted the stimulation frequency at which fused tetanic contractions occurred, independent of age, sex, and muscle (Fig. $2 H$ ). Together, all isometric twitch and tetanic speed parameters steadily and significantly increased over vocal development in male songbirds.

\section{Supralinear force summation increases with muscle speed} Activating muscle fibers with multiple spikes at sufficiently small ISIs leads to summed force responses that are larger than expected from simple algebraic summation of single twitches (Kernell, 1995), which is an intrinsic property of the NMT, often referred to as supralinear or nonlinear force summation. If supralinear force summation occurs, the force-ISI relationship cannot be described by a simple linear regression (Kernell,
1995; Binder et al., 2011; Srivastava et al., 2017). In the songbird respiratory system, millisecond changes in spike timing can cause significant behavioral changes, that reflect 4 and potentially even exploit these nonlinear properties of muscles (Srivastava et al., 2017). The same could be true in syrinx muscles, but we do not know whether they also exhibit supralinear summation to begin with and whether its magnitude changes depending on muscle speed. Therefore, we quantified the magnitude of supralinear force summation in syringeal muscles (see Materials and Methods).

First, when driven by identical stimulation patterns, the shape of force trajectories changed dramatically with muscle contraction speed (Fig. $3 A$ ). $\mathrm{F}_{\max }$ decreased significantly with increasing ISIs (Fig. $3 B$ ) in all preparations and was significantly higher in females than in males for all ISIs tested (females: $2.3 \pm 0.4, N=4$; males: $1.6 \pm 0.4, N=8$ ). Second, force summation was supralinear in all preparations, as the observed force response had a higher $\mathrm{F}_{\max }$ than expected from a summation model (Fig. 3E; see Materials and Methods). The magnitude of supralinear summation significantly depended on spike timing (Fig. 3E), corroborating previous findings in songbird respiratory muscles (Srivastava et al., 2017). Third, the maximal magnitude of supralinear summation increased with muscle speed, regardless of whether it was calculated from $\mathrm{F}_{\max }$ (Fig. $3 F$ ) or AUC (Fig. $3 G$ ). Thus, together, the maximal magnitude of supralinear summation increases with speed and can thus also be expected to increase over development.

\section{Submillisecond changes in spike timing affect magnitude and timing of maximal force development}

The timing of spikes relative to a behavior usually exhibits variability (Stein et al., 2005; Lisberger and Medina, 2015), and it has recently become apparent that the exact timing carries information (Stein et al., 2005; Sober et al., 2018). In songbirds, variability in RA indeed correlates to acoustic variability down to the millisecond scale (Sober et al., 2008; Tang et al., 2014). However, in RA firing precision is even higher and is reported as $0.2 \mathrm{~ms}$ (Chi and Margoliash, 2001), but it is unknown whether submillisecond precision spike timing can pass through the syringeal muscle NMT and cause changes in force trajectories.

To address this question, we performed muscle stimulations with three-spike patterns introducing timing changes to the middle spike in steps from 1 to $0.1 \mathrm{~ms}$ (see Materials and Methods). These timing manipulations evoked changes in both the timing and amplitude of force profiles, and the effect differed between stimulation frequencies (Fig. 4A). To quantify the amount of variability introduced by the timing manipulations, we calculated the $\mathrm{CV}_{\text {Timing }}$ and $\mathrm{CV}_{\text {Amplitude }}$ of $\mathrm{F}_{\text {max }}$. Both $\mathrm{CV}_{\text {Timing }}$ and $\mathrm{CV}_{\text {Amplitude were significantly higher at a stimulation frequency }}$ of $200 \mathrm{~Hz}$ compared with $800 \mathrm{~Hz}$ (Fig. 4B,C). While $200 \mathrm{~Hz}$ stimulation patterns caused variability in both the timing and magnitude of $F_{\max }$, higher stimulation frequencies almost exclusively affected $\mathrm{F}_{\max }$ amplitude (Fig. $4 B$ ), but not its timing (Fig. $4 C$ ).

Because the variability of force timing and amplitude introduced by changes in spike timing showed a wide variation between animals, we hypothesized that the effects could depend 
on muscle speed. To assess this hypothesis, we tested for the dependency of $\mathrm{CV}_{\text {Timing }}$ and $\mathrm{CV}_{\text {Amplitude }}$ on $f_{\text {fused }}$, representing the intrinsic contraction speed of each animal. Overall, $\mathrm{CV}_{\text {Amplitude }}$ and $\mathrm{CV}_{\text {Timing }}$ increased significantly with $f_{\text {fused }}$ (Fig. $4 B$ ). However, as the stimulation frequency clearly influenced the magnitude of the CVs as well as the dependency on $f_{\text {fused }}$, we calculated linear models for each stimulation frequency separately. $\mathrm{CV}_{\text {Amplitude }}$ significantly depended on $f_{\text {fused }}$ at 600 and $800 \mathrm{~Hz}$, and on $\mathrm{CV}_{\text {Timing }}$ at $200 \mathrm{~Hz}$ (Fig. 4C). These results mean that the quality of the effect of changes in spike timing are set by the relationship between stimulation frequency and muscle speed (i.e., $\left.f_{\text {fused }}\right)$ : force timing is affected at stimulation frequencies below the $f_{\text {fused }}$ of adult male muscles (range, $173-343 \mathrm{~Hz}$; median, $277 \mathrm{~Hz}$ ), while force amplitude is affected at all stimulation frequencies. The quality of the effect on the other hand is set by muscle speed confirming our hypothesis, that the variability introduced by spike-timing changes depends on the contraction speed of the muscle. Together, our results show that submillisecond spike timing affects the shape of force profiles. Syringeal muscles can thus resolve spike timing in the submillisecond range. Additionally, muscle speed sets the sensitivity of the muscle to changes in force timing, as illustrated by the increase in variability with increasing muscle speed.

Although spike-timing changes in the submillisecond range affected $F_{\max }$ of force profiles, it is not given that these force changes result in changes in sound production within the physiologically relevant range. For illustration, we estimated the maximal pitch difference caused by stimulating VS with our three pulse patterns (Fig. 4A; see Materials and Methods). We can apply our interpretation from DTB to VS, because both muscles produce similar stress (Adam et al., 2020) and the FWHM ranges of VS (4.3-6.6 ms, $N=5$; see Materials and Methods) and DTB (3.9-5.6 ms; Fig. $2 B$ ) overlap. We estimated an average force difference of $8.15 \pm 6.02 \mathrm{mN}(N=10)$ between the minimal and maximal $F_{\max }$ across all spike-timing stimulation patterns (see Materials and Methods). Based on in vitro measurements of the VS force-to-pitch transform (Adam et al., 2020), this translates into an estimated pitch difference of $217 \pm 160 \mathrm{~Hz}$. Because zebra finch females can detect changes in pitch $<1 \mathrm{~Hz}$ (Lohr and Dooling, 1998), this difference is well within the detectable range. Therefore, we conclude that spike-timing changes in the range of submilliseconds might have behavioral correlates.

Changing spike timing in the high-frequency stimulation patterns $(600$ and $800 \mathrm{~Hz})$ always resulted in a reduction of maximal force compared with the regular stimulation pattern (Fig. 4A). We hypothesized that this decrease was caused by moving spikes into the $t_{\text {Ref }}$ of the muscle, which is $2-5 \mathrm{~ms}$ for most skeletal muscles (Farmer et al., 1960). To estimate the unknown $t_{\text {Ref }}$ of syringeal muscles, we stimulated with two pulses with very short ISIs (Fig. $5 A$; see Materials and Methods). The $t_{\text {Ref }}$ was extremely short, with $0.83 \pm 0.08 \mathrm{~ms}$ for female syrinx muscle and $0.82 \pm 0.17 \mathrm{~ms}$ for male syrinx muscle (females, $N=4$; males, $N=9$ ), and was three to seven times faster than regular skeletal muscle (Fig. 5C). Thus, despite the extremely short $t_{\text {Ref, we con- }}$ firmed our hypothesis that the reduction of maximal force due to spike-timing changes at 600 and $800 \mathrm{~Hz}$ is caused by moving spikes into the refractory period of the muscle.

\section{Discussion}

We show that the songbird syrinx undergoes essential transformations during song learning that drastically change how neural commands are translated into force profiles and thereby acoustic features. All isometric contraction speed-related features of male syrinx muscles, such as FWHM, $f_{\text {fused, }}$ and $f_{\text {Tet }}$, significantly increased over development. Interestingly, the latency from stimulus to force production also significantly decreased. Furthermore, we show that two additional NMT features change significantly with muscle speed. First, we establish that supralinear summation of twitch force occurs in syrinx muscles at ISIs in the millisecond range and that its magnitude increases with muscle speeds. Second, we show that spike timing in the submillisecond range can be resolved by syringeal muscles. Force amplitude was affected at all spike rates tested, while changes in peak force timing were only evoked at spike rates below $f_{\text {fused }}$. Furthermore, the sensitivity to changes in spike timing was set by muscle speed. Together, our data provide strong support for our hypothesis that muscle properties relevant to the NMT change over vocal development. The observed NMT changes are driven by syringeal muscle speed as it increases over vocal development. Furthermore, our data show that the extreme speed of adult muscles is not available to juveniles during song learning.

Several mechanisms could underlie the increasing scontraction speed over development in males. First, the speed increase correlates with a shift in myosin heavy chain isoform composition within the entire muscle toward increased expression of the isoform known as superfast myosin, encoded by the MYH13 gene (Mead et al., 2017). Second, the speed increase over development in males could be caused by a change in fiber type, as described in many other systems (Schiaffino and Reggiani, 2011). Syrinx muscles contain two distinct fiber types (fast and superfast) that are present at different ratios in males and females (Christensen et al., 2017). In adult male zebra finches, syrinx muscles have four times more superfast fibers (up to 87\%) and four times fewer fast oxidative fibers than in females (Christensen et al., 2017; Mead et al., 2017). Furthermore, syringeal muscles of adult female zebra finches contract slower (Elemans et al., 2008) and express less MYH13 (Mead et al., 2017). Our data show that at 25 DPH male and female muscle performance was indistinguishable and female muscle speed did not change over development. Together, these findings strongly suggest that myosin genesis underlies the increase in contraction speed, but the causal relationship remains to be established. Furthermore, it remains unknown whether myosin composition changes occur within muscle fibers or between fiber types.

In other motor systems, MYH13 expression is driven by innervation. Experimental transnervation with faster spiking nerves increases muscle speed (Paniello et al., 2001), and increased use is associated with higher MYH13 expression in extraocular muscles (Brueckner et al., 1999; Moncman et al., 2011). Thus, an increase in $\mathrm{MN}$ discharge rate might be driving the upregulation of $\mathrm{MYH13}$ and consequently the increase in contraction speed in syringeal muscles (Adam and Elemans, 2019). Whether this is only due to the increase in high-frequency input to the MNs (Ölveczky et al., 2011) or also because their intrinsic properties change over development is unknown. Understanding what drives the increase in contraction speed thus requires knowing the motor code over development.

The song template is assumed to be fixed during song learning (Mooney, 2009), and individuals are trying to minimize perceptual error in trial-to-trial renditions (Fee and Scharff, 2010). The increasing syringeal muscle speed over development has the consequence that identical motor commands result in distinct force trajectories, depending on postnatal age, and thus alter behavioral output. If birds aim to produce the same force trajectory during song development, we propose they must dynamically 


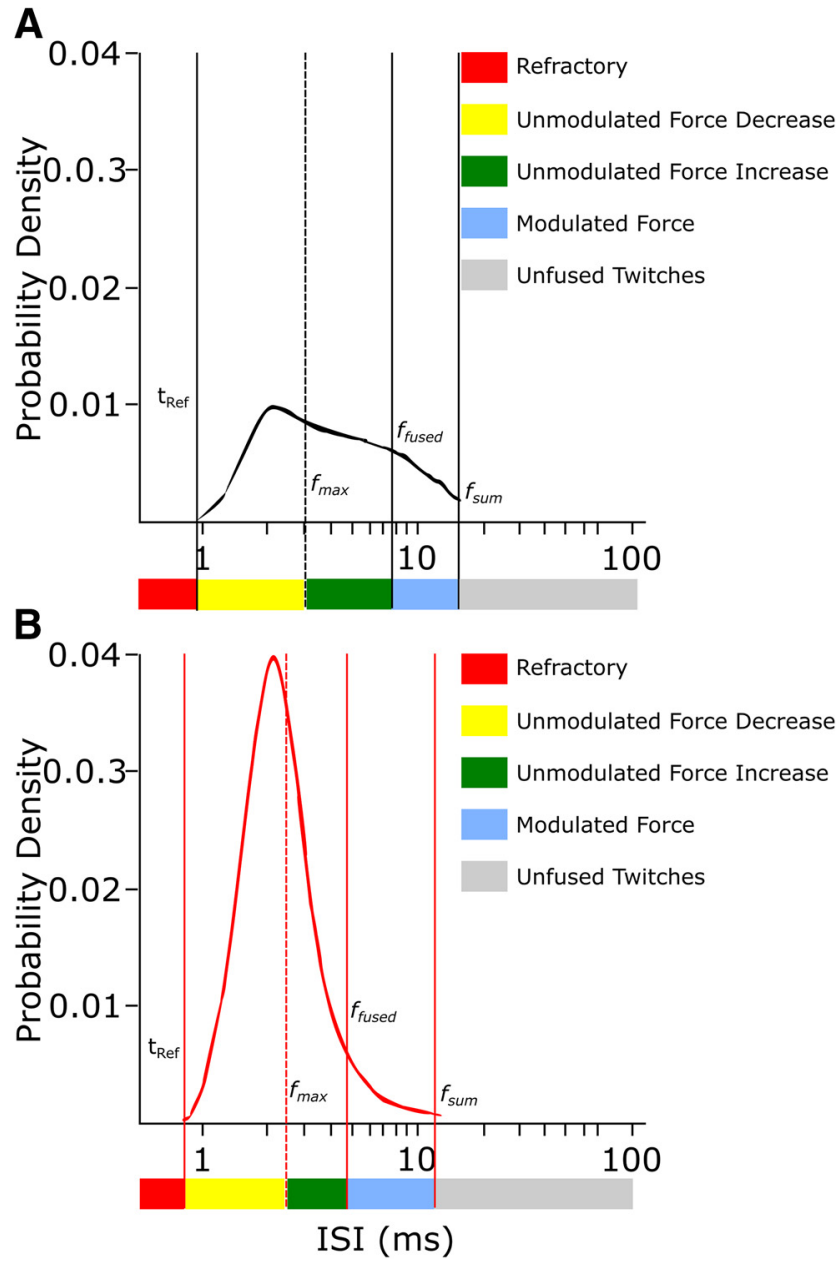

Figure 6. Biomechanical properties of syrinx muscles match the ISI distribution of the last premotor neurons driving them. $\boldsymbol{A}, \boldsymbol{B}$, The $t_{\text {Ref }}$ and summation threshold $\left(f_{\text {sum }}\right)$ of syrinx muscles match the boundaries of the RA-ISI distribution before the start of song learning $(\boldsymbol{A})$ as well as after crystallization (B). The majority of ISIs lie between $t_{\text {Ref }}$ and $f_{\text {fused. }}$. The ISI probability density plots are modified from the study by Ölveczky et al. (2011).

alter their motor code to correct for NMT changes when trying to sing target syllables.

Although the song system neurocircuitry is well studied, the actual motor code, the firing patterns of single units in nXIIts, during singing is unknown (Williams and Nottebohm, 1985; Otchy et al., 2019). In many motor systems, the intrinsic properties of MNs and the biomechanical properties of the innervated muscle fibers are highly tuned (Zhurov and Brezina, 2006; Manuel et al., 2019). For example, the discharge rates of MNs typically fall within the range of frequencies required to generate summed force responses. This is reflected by matching between the firing characteristics of the MN and its muscle FFR, so that the observed range of firing rates modulates forces along the steepest portion of the FFR (Kernell, 1995). This phenomenon is called speed matching. Reversely, speed properties of muscles thus set boundaries within which the motor code is operating. The FFR of a muscle thus allows prediction of the firing rates of the MNs innervating it.

Using our characterization of the biomechanical properties of syringeal muscles, we make three predictions for the nXIIts MN firing frequencies during singing in adult males. First, force summation begins at ISIs $>13.3 \mathrm{~ms}$ (i.e., $75 \mathrm{~Hz}$ ). Thus, to activate syrinx muscles to produce summed force responses, firing rates have to be $>75 \mathrm{~Hz}$. Second, spikes arriving at the NMJ during the refractory period of the muscle will not activate the muscle and are thus lost. To avoid losing spikes, instantaneous firing rates must stay below frequencies leading to lost spikes. Because the refractory period of male syrinx muscles ranges from 0.7 to $1.2 \mathrm{~ms}$, MNs must fire $<1000 \mathrm{~Hz}$. Together, these two predictions set the outer boundaries for motor code spike rates. Last, we predict the peak of the distribution of nXIIts firing frequencies, as follows: force increase by skeletal muscles can be achieved by increasing the number of recruited MNs and thus connected muscle fibers (recruitment), and by increasing the firing rate of MNs (rate coding). Rate coding is most effective on the steep slope of the FFR (Fuglevand et al., 2015; Enoka and Duchateau, 2017). Additionally, to achieve unmodulated force increases, frequencies should be above $f_{\text {fused. }}$. Therefore, we predict ISIs between $3.4 \mathrm{~ms}$ (average $f_{\text {fused }}, 290 \mathrm{~Hz}$ ) and $2.5 \mathrm{~ms}$ (average $f_{\text {Tet }}, 413 \mathrm{~Hz}$ ) to be most suited to change the force output of syrinx muscles in adult males.

If speed matching occurs during the process of vocal development, the ISI distribution of MNs in juveniles should be shifted toward higher ISIs to match the slower speed of the juvenile syringeal muscles. Because of the lack of data on MN firing, we cannot test this hypothesis directly. However, the firing characteristics of the cortical premotor neurons projecting to the MNs in nXIIts (namely $\mathrm{RA}_{\mathrm{nXIIt}}$ ) have been characterized over development (Ölveczky et al., 2011). Interestingly, the refractory period, $f_{\text {Tet }}, f_{\text {fused }}$, and the frequency when force responses start to sum before and after song learning align well with the ISI distributions of age-matched $\mathrm{RA}_{\mathrm{nXIIts}}$ neurons during singing (Fig. 6). The ISI distribution of spikes in RA becomes narrower from subsong (Fig. 6A) to adult song (Fig. 6B), and the boundaries are perfectly demarcated by $t_{\text {Ref }}$ and the frequency when force responses start to sum. The majority of $\mathrm{RA}_{\text {nXIIts }}$ ISIs lie between $t_{\text {Ref }}$ and $f_{\text {fused }}$ for both age groups. Together, RA activity supports the notion that nXIIts MNs and syrinx muscles are speed matched over development.

Interestingly, the refractory period of syrinx muscles $(<1 \mathrm{~ms})$ is shorter than the $t_{\text {Ref }}$ of normal skeletal muscles and within the range of refractory times typically found in MNs (Boërio et al., 2005). We show that syringeal muscle is able to support instantaneous firing rates up to $1000 \mathrm{~Hz}$, while normal skeletal muscles are typically driven by $<50 \mathrm{~Hz}$ (Enoka and Duchateau, 2017). The only muscle activation rates we are aware of that are higher are found in the electric organ of weakly electric fish (Apteronotus leptorhynchus). The electrical organ contains heavily modified myofibers that do not contract, and spinal neurons innervating them can reach brief bursts $>1100 \mathrm{~Hz}$ (Dunlap et al., 2010). Thus, in addition to speed-limiting fundamental architectural constraints of skeletal muscle (Mead et al., 2017), extreme speed in songbird syrinx muscles may additionally be limited by another hard constraint: the refractory time of the CNS.

Our results support the idea that it is critical to include body biomechanics when interpreting motor commands (Nishikawa et al., 2007; Tytell et al., 2011) and emphasize the need for an embodied view of song motor learning (Düring and Elemans, 2016; Zhang and Ghazanfar, 2018). The increased higher variability of juvenile vocalizations (Tchernichovski et al., 2001; Ölveczky et al., 2011) could for example be partially explained by a mismatch between contraction speed and neural firing characteristics during the process of vocal development (Adam and Elemans, 2019; Kollmorgen et al., 2020). Which mechanisms drive muscle speed changes over developmental timescales 
remain unknown, but we recently argued it may be driven predominantly by use (Adam and Elemans, 2019). This implies that extensive training of syringeal muscles is required to achieve maximal speed, and that the total duration and trajectory of song learning may not just be set by neural circuit formation but is also required for extensive muscle training.

\section{References}

Adam I, Elemans CPH (2019) Vocal motor performance in birdsong requires brain-body interaction. eNeuro 6:ENEURO.0053-19.2019.

Adam I, Maxwell A, Rössler H, Hansen EB, Vellema M, Elemans CPH (2020) One-to-one innervation of vocal muscles allows precise control of birdsong. bioRxiv. Advance online publication. Retrieved June 2, 2020.

Binder MD, Heckman CJ, Powers RK (2011) The physiological control of motoneuron activity. In: Rowell LB, Shepherd JT, editors. Handbook of Physiology, p. 3-53. New York, NY: Oxford University Press.

Boërio D, Hogrel JY, Créange A, Lefaucheur JP (2005) A reappraisal of various methods for measuring motor nerve refractory period in humans. Clin Neurophysiol 116:969-976.

Brueckner JK, Ashby LP, Prichard JR, Porter JD (1999) Vestibulo-ocular pathways modulate extraocular muscle myosin expression patterns. Cell Tissue Res 295:477-484.

Chi Z, Margoliash D (2001) Temporal precision and temporal drift in brain and behavior of zebra finch song. Neuron 32:899-910.

Chiel HJ, Beer RD (1997) The brain has a body: adaptive behavior emerges from interactions of nervous system, body and environment. Trends Neurosci 20:553-557.

Christensen LA, Allred LM, Goller F, Meyers RA (2017) Is sexual dimorphism in singing behavior related to syringeal muscle composition? Auk 134:710-720.

Dunlap KD, DiBenedictis BT, Banever SR (2010) Chirping response of weakly electric knife fish (Apteronotus leptorhynchus) to low-frequency electric signals and to heterospecific electric fish. J Exp Biol 213:22342242.

Düring DN, Elemans CPH (2016) Embodied motor control of avian vocal production. In: Vertebrate sound production and acoustic communication (Suthers RA, Fitch WT, Fay RR, Popper AN, eds), pp 119-157. Cham, Switzerland: Springer International.

Elemans CP (2014) The singer and the song: the neuromechanics of avian sound production. Curr Opin Neurobiol 28:172-178.

Elemans CP, Spierts IL, Müller UK, Van Leeuwen JL, Goller F (2004) Bird song: superfast muscles control dove's trill. Nature 431:146.

Elemans CP, Mead AF, Rome LC, Goller F (2008) Superfast vocal muscles control song production in songbirds. PLoS One 3:e2581.

Elemans CP, Rasmussen JH, Herbst CT, During DN, Zollinger SA, Brumm H, Srivastava K, Svane N, Ding M, Larsen ON, Sober SJ, Sõvec JG (2015) Universal mechanisms of sound production and control in birds and mammals. Nat Commun 6:8978.

Enoka RM, Duchateau J (2017) Rate coding and the control of muscle force. Cold Spring Harb Perspect Med 7:a029702.

Farmer TW, Buchthal F, Rosenfalck P (1960) Refractory period of human muscle after the passage of a propagated action potential. Electroencephalogr Clin Neurophysiol 12:455-466.

Fee MS, Scharff C (2010) The songbird as a model for the generation and learning of complex sequential behaviors. ILAR J 51:362-377.

Fitch WT, Huber L, Bugnyar T (2010) Social cognition and the evolution of language: constructing cognitive phylogenies. Neuron 65:795-814.

Fuglevand AJ, Lester RA, Johns RK (2015) Distinguishing intrinsic from extrinsic factors underlying firing rate saturation in human motor units. J Neurophysiol 113:1310-1322.

Goller F, Suthers RA (1996) Role of syringeal muscles in controlling the phonology of bird song. J Neurophysiol 76:287-300.

Hahnloser RH, Kozhevnikov AA, Fee MS (2002) An ultra-sparse code underlies the generation of neural sequences in a songbird. Nature 419:65-70.

Immelmann K (1984) The natural history of bird learning. In: The biology of learning. Dahlem Workshop Reports (Life Sciences Research Reports), Vol 29 (Marler P, Terrace HS, eds). Berlin, Heidelberg: Springer.

Jarvis ED (2004) Learned birdsong and the neurobiology of human language. Ann N Y Acad Sci 1016:749-777.
Kernell D (1995) Neuromuscular frequency-coding and fatigue. Adv Exp Med Biol 384:135-145.

Kollmorgen S, Hahnloser RHR, Mante V (2020) Nearest neighbours reveal fast and slow components of motor learning. Nature 577:526-530.

Leonardo A, Fee MS (2005) Ensemble coding of vocal control in birdsong. J Neurosci 25:652-661.

Lisberger SG, Medina JF (2015) How and why neural and motor variation are related. Curr Opin Neurobiol 33:110-116.

Lohr B, Dooling RJ (1998) Detection of changes in timbre and harmonicity in complex sounds by zebra finches (Taeniopygia guttata) and budgerigars (Melopsittacus undulatus). J Comp Psychol 112:36-47.

Manuel M, Chardon M, Tysseling V, Heckman CJ (2019) Scaling of motor output, from mouse to humans. Physiology (Bethesda) 34:5-13.

Mead AF, Osinalde N, Ørtenblad N, Nielsen J, Brewer J, Vellema M, Adam I, Scharff C, Song Y, Frandsen U, Blagoev B, Kratchmarova I, Elemans CP (2017) Fundamental constraints in synchronous muscle limit superfast motor control in vertebrates. Elife 6:e29425.

Mendez J, Keys A (1960) Density and composition of mammalian muscle. Metabolism 9:184-188.

Moncman CL, Andrade ME, Andrade FH (2011) Postnatal changes in the developing rat extraocular muscles. Invest Ophthalmol Vis Sci 52:39623969.

Mooney R (2009) Neural mechanisms for learned birdsong. Learn Mem 16:655-669.

Nishikawa K, Biewener AA, Aerts P, Ahn AN, Chiel HJ, Daley MA, Daniel TL, Full RJ, Hale ME, Hedrick TL, Lappin AK, Nichols TR, Quinn RD, Satterlie RA, Szymik B (2007) Neuromechanics: an integrative approach for understanding motor control. Integr Comp Biol 47:16-54.

Nottebohm F, Stokes TM, Leonard CM (1976) Central control of song in the canary, Serinus canarius. J Comp Neurol 165:457-486.

Ölveczky BP, Otchy TM, Goldberg JH, Aronov D, Fee MS (2011) Changes in the neural control of a complex motor sequence during learning. J Neurophysiol 106:386-397.

Orizio C, Gobbo M, Diemont B (2004) Changes of the force-frequency relationship in human tibialis anterior at fatigue. J Electromyogr Kinesiol 14:523-530.

Otchy TM, Michas C, Lee B, Gopalan K, Gleick J, Semu D, Darkwa L, Holinski BJ, Chew DJ, White AE, Gardner TJ (2019) Printable microscale interfaces for long-term peripheral nerve mapping and precision control. bioRxiv. Advance online publication. Retrieved June 2, 2020.

Paniello RC, West SE, Lee P (2001) Laryngeal reinnervation with the hypoglossal nerve. I. Physiology, histochemistry, electromyography, and retrograde labeling in a canine model. Ann Otol Rhinol Laryngol 110:532-542.

Raikova RT, Aladjov H (2002) Hierarchical genetic algorithm versus static optimization-investigation of elbow flexion and extension movements. J Biomech 35:1123-1135.

Riede T, Goller F (2010) Peripheral mechanisms for vocal production in birds-differences and similarities to human speech and singing. Brain Lang 115:69-80.

Ritz C, Baty F, Streibig JC, Gerhard D (2015) Dose-response analysis using R. PLoS One 10:e0146021.

Roper A, Zann R (2006) The onset of song learning and song tutor selection in fledgling zebra finches. Ethology 112:458-470.

Scharff C, Nottebohm F (1991) A comparative study of the behavioral deficits following lesions of various parts of the zebra finch song system: implications for vocal learning. J Neurosci 11:2896-2913.

Schiaffino S, Reggiani C (2011) Fiber types in mammalian skeletal muscles. Physiol Rev 91:1447-1531.

Signorell A (2019) DescTools: tools for descriptive statistics. Vienna: R Foundation for Statistical Computing.

Sober SJ, Wohlgemuth MJ, Brainard MS (2008) Central contributions to acoustic variation in birdsong. J Neurosci 28:10370-10379.

Sober SJ, Sponberg S, Nemenman I, Ting LH (2018) Millisecond spike timing codes for motor control. Trends Neurosci 41:644-648.

Srivastava KH, Elemans CP, Sober SJ (2015) Multifunctional and context-dependent control of vocal acoustics by individual muscles. J Neurosci 35:14183-14194.

Srivastava KH, Holmes CM, Vellema M, Pack AR, Elemans CPH, Nemenman I, Sober SJ (2017) Motor control by precisely timed spike patterns. Proc Natl Acad Sci U S A 114:1171-1176. 
Stein RB, Gossen ER, Jones KE (2005) Neuronal variability: noise or part of the signal? Nat Rev Neurosci 6:389-397.

Tang C, Chehayeb D, Srivastava K, Nemenman I, Sober SJ (2014) Millisecondscale motor encoding in a cortical vocal area. PLoS Biol 12:e1002018.

Tchernichovski O, Mitra PP, Lints T, Nottebohm F (2001) Dynamics of the vocal imitation process: how a zebra finch learns its song. Science 291:2564-2569.

Tytell ED, Holmes P, Cohen AH (2011) Spikes alone do not behavior make: why neuroscience needs biomechanics. Curr Opin Neurobiol 21:816-822.

Watanabe S, Kitawaki T, Oka H (2010) Mathematical equation of fusion index of tetanic contraction of skeletal muscles. J Electromyogr Kinesiol 20:284-289.

Wild JM (1993a) The avian nucleus retroambigualis: a nucleus for breathing, singing and calling. Brain Res 606:319-324.

Wild JM (1993b) Descending projections of the songbird nucleus robustus archistriatalis. J Comp Neurol 338:225-241.
Wild JM (1997) Neural pathways for the control of birdsong production. J Neurobiol 33:653-670.

Williams H, Nottebohm F (1985) Auditory responses in avian vocal motor neurons: a motor theory for song perception in birds. Science 229:279282.

Zann RA (1996) The zebra finch: a synthesis of field and laboratory studies. Oxford, UK: Oxford UP.

Zhang YS, Ghazanfar AA (2018) Vocal development through morphological computation. PLoS Biol 16:e2003933.

Zhang YS, Takahashi DY, Liao DA, Ghazanfar AA, Elemans CPH (2019) Vocal state change through laryngeal development. Nat Commun 10:4592.

Zhurov Y, Brezina V (2006) Variability of motor neuron spike timing maintains and shapes contractions of the accessory radula closer muscle of Aplysia. J Neurosci 26:7056-7070. 